\title{
Estresse e sobrepeso/obesidade em estudantes de enfermagem
}

\author{
Janete de Souza Urbanetto ${ }^{1}$ \\ (1D) https://orcid.org/0000-0002-4697-1641 \\ Pâmela Silva da Rocha² \\ (iD) https://orcid.org/0000-0003-3551-9331 \\ Rosangela Carvalho Dutra ${ }^{3}$ \\ (1D) https://orcid.org/0000-0001-8734-7768 \\ Maria Carolina Maciel ${ }^{1}$ \\ (1D) https://orcid.org/0000-0001-9584-6487 \\ Andrea Gonçalves Bandeira ${ }^{1}$ \\ (D) https://orcid.org/0000-0002-0794-8027 \\ Tania Solange Bosi de Souza Magnago 4 \\ (D) https://orcid.org/0000-0002-5308-1604
}

\begin{abstract}
Objetivo: analisar a associação entre características demográficas, acadêmicas, de saúde, estresse, sobrepeso e obesidade em estudantes de enfermagem. Método: estudo transversal, com 95 estudantes de uma universidade privada do Rio Grande do Sul, Brasil. Aplicou-se questionário de caracterização demográfica, acadêmica e de saúde e utilizouse a Escala de Avaliação do Estresse em Estudantes de Enfermagem. Medidas antropométricas foram mensuradas. Empregaram-se análises descritivas e bivariadas. Resultados: predominaram estudantes do sexo feminino, com idade média de 25,6 \pm 5,87 anos. Observou-se aumento de peso em 52,6\% dos estudantes e níveis de estresse alto $(29,5 \%)$ e muito alto $(36,8 \%)$ no domínio Formação Profissional. Nenhum dos domínios da escala de estresse se mostrou associado ao sobrepeso e obesidade. Conclusão: o sobrepeso e obesidade mostraram-se associados ao sexo masculino, pressão arterial elevada, ganho de peso desde o início do curso, circunferência abdominal alterada, não realização de atividade física, comer mais em situações de estresse e ingerir alimentos não saudáveis.
\end{abstract}

Descritores: Estudantes de Enfermagem; Enfermagem; Estresse Psicológico; Obesidade; Sobrepeso; Estilo de Vida.

\section{Como citar este artigo}

Urbanetto JS, Rocha PS, Dutra RC, Maciel MC, Bandeira AG, Magnago TSBS. Stress and overweight/ obesity among nursing students. Rev. Latino-Am. Enfermagem. 2019;27:e3177. [Access Available in: DOI: http://dx.doi.org/10.1590/1518-8345.2966.3177. mes día año 


\section{Introdução}

Nas últimas décadas, a obesidade tem sido relacionada às mudanças sócio comportamentais, ao consumo de alimentos calóricos, de alta palatabilidade e baixo poder sacietógeno, bem como ao aumento do conteúdo calórico de cada refeição e nas alimentações feitas fora de casa, em redes de fast food(1). Ela é considerada um agravo de caráter multifatorial, que envolve questões biológicas, históricas, ecológicas, econômicas, sociais, culturais e políticas ${ }^{(2)}$.

Segundo a Associação Brasileira para o Estudo da Obesidade e da Síndrome Metabólica (ABESO), o estresse psicológico pode contribuir para o ganho de peso e obesidade, considerando que a ansiedade pode levar ao aumento da ingestão de alimentos, ao controle inadequado do apetite e à compulsão alimentar(1). Ele é, também, um agravo complexo pela sua multicausalidade e se dá na interação do indivíduo com seu meio ${ }^{(3-4)}$. Essa influência mútua pode ocasionar alterações físicas, psíquicas, emocionais e comportamentais ${ }^{(4)}$. Estudo que avaliou a síndrome do comer noturno evidenciou que estudantes universitários brasileiros possuem elevada prevalência desse comportamento, o qual mostrou-se associado a sintomas depressivos, de ansiedade e estresse ${ }^{(5)}$

Na prática diária, o estresse pode se manifestar por comportamentos observáveis ${ }^{(3)}$. Os estressores podem surgir em vários momentos, a partir de pressões relacionadas à vida pessoal, social, profissional e, também, na vida acadêmica. Nesta última, a vivência de mudanças, desenvolvimento, temores e angústia, entre outras, podem funcionar como desencadeadores de estresse e de agravos na saúde(6).

Estudantes de enfermagem consideram estressores o medo do desconhecido, a sobrecarga em atividades acadêmicas, os sentimentos de insegurança e impotência(7), a insatisfação com o curso e o excesso de atividades ${ }^{(8)}$. Também relataram como estressores a sobrecarga e o aumento da responsabilidade no último ano do curso; apontaram que a elaboração do trabalho de conclusão de curso, as preocupações relacionadas à inserção no mercado de trabalho, a relação trabalho/estudo/vida familiar e as dificuldades nos relacionamentos interpessoais são situações causadoras de desgaste físico e angústia(9).

Nesse contexto, é possível que as exigências da vida acadêmica e a incerteza da vida profissional que se aproxima produzam importante carga emocional nos estudantes, alterando seus comportamentos e estilo de vida. De acordo com estudantes da área da saúde, as atividades acadêmicas, além de causar estresse, estimulam o estudante a buscar alimentos fora do domicilio ${ }^{(10)}$. A médio e longo prazo, sem critérios de uma dieta equilibrada, esse comportamento pode contribuir para o aumento de peso nos estudantes.

Com base no exposto, identificou-se a necessidade de explorar mais essa temática, corroborando na identificação dos fatores relacionados ao sobrepeso/ obesidade em estudantes universitários, bem como fornecer subsídios para o enfrentamento dessa situação. As questões de pesquisa estabelecidas para este estudo foram: Quais são os fatores associados ao sobrepeso ou obesidade em estudantes de enfermagem? O estresse está associado ao sobrepeso ou obesidade em estudantes de enfermagem? Para responder a essas questões, foi estabelecido como objetivo analisar a associação entre características demográficas, acadêmicas, de saúde, estresse, sobrepeso e obesidade em estudantes de enfermagem.

\section{Método}

Trata-se de um estudo transversal, aninhado a um projeto de pesquisa com delineamento de coorte intitulado "Avaliação da ocorrência de dor musculoesquelética e distúrbios psíquicos menores em estudantes de enfermagem", realizado em um curso de Graduação em Enfermagem de uma universidade privada do Rio Grande do Sul, Brasil.

A população do estudo foi composta por 144 estudantes do referido curso de Enfermagem. Para o cálculo amostral, considerou-se um percentual estimado de $0,5 \%$, intervalo de confiança de $95 \%$ e um erro amostral de 0,05\%. A partir desse cálculo, para garantir a representatividade, a amostra mínima seria de 89 estudantes, no entanto, todos os 114 estudantes foram convidados a participar do estudo. Como critérios de inclusão, os estudantes deveriam estar regularmente matriculados e ter idade $\geq 18$ anos. Foram excluídos aqueles que estavam afastados por qualquer motivo das atividades acadêmicas (licença, mobilidade, trancamento parcial ou total do curso).

A coleta de dados foi realizada entre setembro e dezembro de 2016, de forma não probabilística (todos foram convidados), conforme agendamento com os estudantes. As variáveis coletadas foram: a) demográficas: sexo (feminino e masculino), idade (em anos completos e, em seguida, dicotomizada em $\leq 26$ anos e $\geq 27$ anos), situação conjugal (solteiros; casados/união estável), cor da pele (branca; preta e parda), e se possui filhos (sim; não); b) acadêmicas: semestre e ano de ingresso no curso; c) de saúde: peso $(\mathrm{kg})$, altura $(\mathrm{cm})$, circunferência abdominal $(\mathrm{cm})$, pressão arterial $(\mathrm{mmHg})$, realização de atividade física (não; sim; às vezes), e questões que avaliam os 
hábitos alimentares dos estudantes ${ }^{(11)}$; d) estresse dos estudantes, por meio da Escala de Avaliação do Estresse em Estudantes de Enfermagem (AEEE) (3).

A Escala AEEE é composta por 30 questões que avaliam a intensidade de estresse. Possui quatro opções de resposta para cada item ( 0 = não vivencio a situação; 1 = não me sinto estressado com a situação; 2 = me sinto pouco estressado com a situação; 3 = me sinto muito estressado com a situação). A escala também possui seis domínios: Realização de Atividades Práticas; Comunicação Profissional; Gerenciamento do Tempo; Ambiente; Formação Profissional e Atividade Teórica(3).

O Domínio 1 - Realização de Atividades Práticas é composto por seis itens que se referem ao conhecimento instrumental adquirido pelo aluno para a realização dos procedimentos e os sentimentos envolvidos na oferta do cuidado ao paciente. No Domínio 2 - Comunicação Profissional, os quatro itens retratam as dificuldades sentidas na comunicação e na relação do indivíduo com os elementos do convívio profissional e as situações conflitantes que surgem. 0 Domínio 3 - Gerenciamento do Tempo, com cinco itens, considera as dificuldades relatadas pelos estudantes para conciliar as atividades estabelecidas na grade curricular com as exigências pessoais, emocionais e sociais. No Domínio 4 - Ambiente, os quatro itens retratam o grau de dificuldade sentido no acesso aos campos de estágio ou universidade e as situações de desgaste percebidas pelos estudantes com os meios de transportes utilizados. Os seis itens do Domínio 5 - Formação Profissional se referem à preocupação do aluno sobre o conhecimento adquirido em sua fase de formação acadêmica e o impacto que este exerce sobre sua futura vida profissional. Inclui, ainda, a percepção das situações que poderá vivenciar quando profissional. Os cinco itens do Domínio 6 - Atividade Teórica se referem ao grau de dificuldade sentido pelos estudantes com o conteúdo programático, às atividades desenvolvidas e à metodologia de ensino adotada(3).

A classificação dos níveis de estresse foi realizada por domínio, por meio da soma das pontuações das questões equivalentes a cada um: Domínio 1: 0-9 baixo nível de estresse; $10-12$ médio nível de estresse; 13-14 alto nível de estresse; 15-18 muito alto nível de estresse. Domínio 2: 0-5 baixo nível de estresse; 6 médio nível de estresse; 7-8 alto nível de estresse; 9-12 muito alto nível de estresse. Domínio 3: 0-10 baixo nível de estresse; 11-12 médio nível de estresse; 13-14 alto nível de estresse; 15 muito alto nível de estresse. Domínio 4: 0-7 baixo nível de estresse; 8-10 médio nível de estresse; 11 alto nível de estresse; 12 muito alto nível de estresse. Domínio 5: 0-9 baixo nível de estresse; 10 médio nível de estresse; 11-12 alto nível de estresse; $13-18$ muito alto nível de estresse. Domínio 6: 0-9 baixo nível de estresse; 10-11 médio nível de estresse; $12-13$ alto nível de estresse; 14-15 muito alto nível de estresse(3).

A pressão arterial (PA) foi verificada duas vezes conforme a $7^{a}$ Diretriz Brasileira de Hipertensão: braço posicionado na altura do coração, apoiado, com a palma da mão voltada para cima, atentando para que as roupas não garroteassem o membro e utilizando manguito adequado à circunferência do braço, com o estudante sentado, com as pernas descruzadas, pés apoiados no chão, dorso recostado na cadeira e relaxado(12).

A primeira verificação ocorreu com o estudante em repouso por 5 a 10 minutos em ambiente calmo, certificando se o estudante não estava com a bexiga cheia, não havia praticado exercícios físicos há pelo menos 60 minutos, não houvesse ingerido bebidas alcoólicas, café ou alimentos ou fumado nos 30 minutos anteriores. A segunda verificação foi realizada após o preenchimento do questionário, em torno de 20 minutos de diferença entre a primeira e a segunda verificação. O equipamento usado nas medidas era digital, com certificação, calibrado, mantido e transportado adequadamente.

Para a classificação da PA utilizou-se a recomendação da 7a Diretriz Brasileira de Hipertensão, considerando-se hipertensos os estudantes com valor de PA sistólica (PAS) $\geq 140 \mathrm{mmHg}$ e/ou PA diastólica (PAD) $\geq 90 \mathrm{mmHg}$, obtidas pela média dos valores encontrados na primeira e na segunda verificação(12).

O peso no início do curso de graduação foi autorreferido e o atual foi verificado com balança digital. A diferença entre eles foi dicotomizada pela mediana em função da distribuição anormal (Shapiro-Wilk = 0,022). A altura foi medida com o auxílio de um estadiômetro. Para o cálculo do Índice de Massa Corpórea (IMC), aplicou-se a fórmula IMC=peso/altura ${ }^{2}$. Para classificação do IMC, foram utilizados os pontos de corte de $18,5-24,9 \mathrm{~kg} / \mathrm{m}^{2}$ (eutrofia), 25 a $29,9 \mathrm{~kg} / \mathrm{m}^{2}$ (sobrepeso) e $\geq 30 \mathrm{~kg} / \mathrm{m}^{2}$

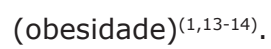

Para identificação da obesidade central, foi utilizado a medida da circunferência abdominal, colocando-se a fita métrica no ponto médio, entre o rebordo costal inferior e a borda superior da crista ilíaca, com o participante em posição ortostática. Considerando os pontos de corte da circunferência abdominal $(90 \mathrm{~cm}$ para homens e $80 \mathrm{~cm}$ para mulheres) e a origem étnica recomendada pela International Diabetes Federation(15) e pela $7^{a}$ Diretriz Brasileira de Hipertensão Arterial(12), a categorização do risco cardiovascular ficou assim determinada: sem risco (circunferência abdominal $<90 \mathrm{~cm}$ para homens e $<80 \mathrm{~cm}$ para mulheres), 
risco aumentado ( $\geq 90 \mathrm{a}<102 \mathrm{~cm}$ para homens e $\geq 80$ a $<88 \mathrm{~cm}$ para mulheres) e risco substancialmente aumentado ( $\geq 102 \mathrm{~cm}$ para homens e $\geq 88 \mathrm{~cm}$ para mulheres)(14).

Após dupla digitação independente no programa Excel e correção de incongruências da digitação, os dados foram analisados no programa PASW Statistics ${ }^{\circledR}$ (Predictive Analytics Software, da SPSS Inc., Chicago Estados Unidos) 18.0 para Windows, utilizando-se da estatística descritiva e inferencial. Os dados quantitativos foram descritos em medidas de tendência central (média ou mediana) e dispersão (desvio padrão, amplitude), conforme sua distribuição de normalidade ou não (Teste Shapiro-Wilk). A verificação de associação entre as variáveis independentes (demográficas, acadêmicas, hábitos e de saúde) e os desfechos (sobrepeso e obesidade) foi medida pelo teste Qui-quadrado ou exato de Fischer (menos de 5 células). Em todas as análises foi considerado o nível de significância de $5 \%$.

O projeto de pesquisa foi aprovado pelo Comitê de Ética da instituição sob registro CAAE 50096615.1.0000.5336. Todos os estudantes que participaram do estudo assinaram o Termo de Consentimento Livre e Esclarecido em duas vias.

\section{Resultados}

Participaram do estudo 95 estudantes (83,3\%) de todos os semestres do curso de graduação em enfermagem de uma universidade privada do Rio Grande do Sul - Brasil. Destes, 34 (35,8\%) estudantes pertenciam ao oitavo semestre, 21 (22,1\%), ao sexto semestre, $18(18,9 \%)$, ao segundo semestre e $17(17,9 \%)$, ao quarto semestre do curso. 19 estudantes $(16,7 \%)$ não aceitaram participar do estudo.

$\mathrm{Na}$ Tabela 1, apresenta-se a classificação dos estudantes quanto ao perfil demográfico, prática de atividade física, Índice de Massa Corporal, níveis pressóricos e circunferência abdominal.

Predominaram estudantes com até 26 anos (25,68 \pm 5,87 anos, com mínimo de 18 anos e máximo de 43 anos), do sexo feminino, autodeclarados brancos, solteiros, sem filhos e que não praticam atividade física. Maior percentual tinha alteração no peso (sobrepeso, obesidade I e II), foram classificados como pré-hipertensos e com risco cardiovascular (Tabela 1).

Quanto ao peso autorreferido no início do curso, a média foi de $68,08 \mathrm{~kg}$. Já o peso médio atual aferido foi de $71,43 \mathrm{~kg}$. Considerando a diferença entre os dois valores, metade dos estudantes tiveram um ganho de $3,55 \mathrm{~kg}$ do início da graduação até o momento da pesquisa. A maior perda de peso foi de $42,3 \mathrm{~kg}$ e o máximo de ganho foi de $28 \mathrm{~kg}$.
Tabela 1 - Caracterização das variáveis demográficas e de saúde dos estudantes de enfermagem. Porto Alegre, RS, Brasil, 2016. $\mathrm{n}=95$ estudantes

\begin{tabular}{|c|c|c|}
\hline Variáveis & $\mathbf{n}$ & $\%$ \\
\hline \multicolumn{3}{|l|}{ Idade } \\
\hline Até 26 anos & 64 & 67,4 \\
\hline 27 anos ou mais & 31 & 32,6 \\
\hline \multicolumn{3}{|l|}{ Sexo } \\
\hline Feminino & 76 & 80,0 \\
\hline Masculino & 19 & 20,0 \\
\hline \multicolumn{3}{|l|}{ Cor da pele autodeclarada $(n=94)$} \\
\hline Branca & 85 & 90,4 \\
\hline Preta & 6 & 6,4 \\
\hline Parda & 3 & 3,2 \\
\hline \multicolumn{3}{|l|}{ Situação conjugal ( $n=93$ ) } \\
\hline Solteiros & 74 & 79,6 \\
\hline Casados/união estável & 19 & 20,4 \\
\hline \multicolumn{3}{|l|}{ Possui filhos $(n=90)$} \\
\hline Sim & 23 & 25,5 \\
\hline Não & 67 & 74,4 \\
\hline \multicolumn{3}{|l|}{ Pratica atividade física } \\
\hline Sim & 17 & 17,9 \\
\hline Não & 43 & 45,3 \\
\hline Às vezes & 35 & 36,8 \\
\hline \multicolumn{3}{|l|}{ Índice de Massa Corporal } \\
\hline Eutrofia & 45 & 47,4 \\
\hline Sobrepeso & 34 & 35,8 \\
\hline Obesidade I & 11 & 11,6 \\
\hline Obesidade II & 5 & 5,2 \\
\hline \multicolumn{3}{|l|}{ Classificação da pressão arterial } \\
\hline Normal & 29 & 30,5 \\
\hline Pré-hipertensão & 42 & 44,2 \\
\hline Hipertensão I & 14 & 14,7 \\
\hline Hipertensão II & 7 & 7,4 \\
\hline Hipertensão III & 3 & 3,2 \\
\hline \multicolumn{3}{|l|}{ Circunferência abdominal (risco cardiovascular - sexo) } \\
\hline$<80 \mathrm{~cm}$ (sem risco - feminino) & 38 & 40,0 \\
\hline < 90 cm (sem risco - masculino) & 7 & 7,4 \\
\hline$\geq 80$ e $<88 \mathrm{~cm}$ (risco aumentado - feminino) & 19 & 20,0 \\
\hline$\geq 90 \mathrm{e}<102 \mathrm{~cm}$ (risco aumentado - masculino) & 10 & 10,5 \\
\hline $\begin{array}{l}\geq 88 \mathrm{~cm} \text { (risco substancialmente aumentado - } \\
\text { feminino) }\end{array}$ & 19 & 20,0 \\
\hline $\begin{array}{l}\geq 102 \mathrm{~cm} \text { (risco substancialmente aumentado - } \\
\text { masculino) }\end{array}$ & 2 & 2,1 \\
\hline
\end{tabular}

Fonte: dados da pesquisa, 2016

Quando questionados se consideravam sua alimentação saudável, pensando na rotina diária, $55(57,9 \%)$ responderam que não e 42 (44,2\%) estudantes afirmam realizar troca das principais refeições do dia por lanches rápidos. Quanto às refeições clássicas 
do dia, 38 (40\%) estudantes realizam o desjejum, 52 $(54,7 \%)$ o lanche da manhã, $90(94,6 \%)$ o almoço, 69 $(72,6 \%)$ o lanche da tarde, $92(86,3 \%)$ o jantar e 21 $(22,1 \%)$ a ceia. Maior percentual de estudantes $(74,7 \%)$ costuma realizar as refeições assistindo TV, mexendo no computador, tablet e/ou celular. Em situações de estresse, $63(66,3 \%)$ estudantes responderam que tendem a sentir mais vontade de comer.
A Tabela 2 apresenta a classificação de estresse (nível baixo, médio, alto ou muito alto) de acordo com os domínios da Escala AEEE.

Na Tabela 3, apresentam-se os dados de associação do IMC com variáveis demográficas, acadêmicas, laborais e de saúde.

$\mathrm{Na}$ Tabela 4, são apresentados os dados da associação do IMC com os domínios da Escala AEEE.

Tabela 2 - Classificação do estresse por domínios da Escala de Estresse nos Estudantes de Enfermagem. Porto Alegre, RS, Brasil, 2016. $\mathrm{n}=95$ estudantes

\begin{tabular}{|c|c|c|c|c|c|c|}
\hline \multirow{3}{*}{ Categorias do estresse } & \multicolumn{6}{|c|}{ Domínios da AEEE* } \\
\hline & $\begin{array}{c}\text { Realização } \\
\text { Atividades } \\
\text { Práticas }\end{array}$ & $\begin{array}{c}\text { Comunicação } \\
\text { Profissional }\end{array}$ & $\begin{array}{l}\text { Gerenciamento } \\
\text { de Tempo }\end{array}$ & Ambiente & $\begin{array}{l}\text { Formação } \\
\text { Profissional }\end{array}$ & $\begin{array}{l}\text { Atividade } \\
\text { Teórica }\end{array}$ \\
\hline & $\mathrm{n}(\%)$ & n (\%) & n (\%) & n (\%) & n (\%) & n (\%) \\
\hline Baixo nível de estresse & $31(32,6)$ & $41(43,2)$ & $60(63,2)$ & $58(61,1)$ & $23(24,2)$ & $42(44,2)$ \\
\hline Médio nível de estresse & $30(31,6)$ & $23(24,2)$ & $11(11,6)$ & $17(17,9)$ & $9(9,5)$ & $27(28,4)$ \\
\hline Alto nível de estresse & $22(23,2)$ & $17(17,9)$ & $18(18,9)$ & $8(8,4)$ & $28(29,5)$ & $16(16,8)$ \\
\hline Muito alto nível de estresse & $12(12,6)$ & $14(14,7)$ & $6(6,3)$ & $12(12,6)$ & $35(36,8)$ & $10(10,5)$ \\
\hline
\end{tabular}

Fonte: dados da pesquisa, 2016. Nota *AEEE = Avaliação de Estresse nos Estudantes de Enfermagem

Tabela 3 - Associação das variáveis demográficas, acadêmicas, laborais e de saúde com o Índice de Massa Corpórea. Porto Alegre, RS, Brasil, 2016. $\mathrm{n}=95$ estudantes

\begin{tabular}{|c|c|c|c|c|c|c|c|}
\hline \multirow{3}{*}{ Variáveis independentes } & \multicolumn{6}{|c|}{ Índice de Massa Corporal* } & \multirow{3}{*}{$\mathbf{p}$} \\
\hline & \multicolumn{2}{|c|}{ Eutrofia } & \multicolumn{2}{|c|}{ Sobrepeso } & \multicolumn{2}{|c|}{ Obesidade } & \\
\hline & $\mathbf{n}$ & $\%$ & $\mathbf{n}$ & $\%$ & $\mathbf{n}$ & $\%$ & \\
\hline \multicolumn{8}{|l|}{ Idade } \\
\hline Até 26 anos & 34 & 53,1 & 23 & 35,9 & 7 & 10,9 & \multirow{2}{*}{$0,066^{\dagger}$} \\
\hline 27 anos ou mais & 11 & 35,5 & 11 & 35,5 & 9 & 29,0 & \\
\hline \multicolumn{8}{|l|}{ Sexo } \\
\hline Feminino & 40 & 52,6 & 22 & 28,9 & 14 & 18,4 & \multirow{2}{*}{$0,032^{\ddagger}$} \\
\hline Masculino & 5 & 26,3 & 12 & 63,2 & 2 & 10,5 & \\
\hline \multicolumn{8}{|l|}{ Variação de peso $(n=90)$} \\
\hline Variação de peso $\leq 3,55 \mathrm{~kg}$ & 32 & 71,1 & 9 & 20,0 & 4 & 8,9 & \multirow{2}{*}{$<0,001^{\ddagger}$} \\
\hline Variação de peso $\geq 3,55 \mathrm{~kg}$ & 12 & 26,7 & 21 & 46,7 & 12 & 26,7 & \\
\hline \multicolumn{8}{|l|}{ Circunferência abdominal } \\
\hline Sem risco & 36 & 80,0 & 9 & 20,0 & - & - & \multirow{3}{*}{$<0,001^{\dagger}$} \\
\hline Risco aumentado & 8 & 27,6 & 20 & 69,0 & 1 & 3,4 & \\
\hline Risco substancialmente aumentado & 1 & 4,8 & 5 & 23,8 & 15 & 71,4 & \\
\hline \multicolumn{8}{|l|}{ Pratica atividade física } \\
\hline Às vezes & 16 & 45,7 & 16 & 45,7 & 3 & 8,6 & \multirow{3}{*}{$0,027^{\ddagger}$} \\
\hline Sim & 7 & 41,2 & 9 & 52,9 & 1 & 5,9 & \\
\hline Não & 22 & 51,2 & 9 & 20,9 & 12 & 27,9 & \\
\hline \multicolumn{8}{|l|}{ Pressão arterial } \\
\hline Normal & 16 & 55,2 & 8 & 27,6 & 5 & 17,2 & \multirow{3}{*}{$0,042^{+}$} \\
\hline Pré-hipertensão & 24 & 57,1 & 13 & 31,0 & 5 & 11,9 & \\
\hline Hipertensão & 5 & 20,8 & 13 & 54,2 & 6 & 25,0 & \\
\hline \multicolumn{8}{|l|}{ Semestre } \\
\hline Até o quarto semestre & 19 & 51,4 & 12 & 32,4 & 6 & 16,2 & \multirow{2}{*}{$0,616^{\dagger}$} \\
\hline Quinto ao oitavo semestre & 26 & 44,8 & 22 & 37,9 & 10 & 17,2 & \\
\hline \multicolumn{8}{|l|}{ Troca de refeições por lanches $(n=93)$} \\
\hline Sim & 15 & 35,7 & 20 & 47,6 & 7 & 16,7 & \multirow{2}{*}{$0,139^{+}$} \\
\hline Não & 29 & 56,9 & 13 & 25,5 & 9 & 17,6 & \\
\hline \multicolumn{8}{|c|}{ Reação em situações de estresse ou ansiedade } \\
\hline Sentir mais vontade de comer & 24 & 38,1 & 25 & 39,7 & 14 & 22,2 & \multirow{2}{*}{$0,025^{\ddagger}$} \\
\hline Sentir menos vontade de comer & 21 & 65,6 & 9 & 28,1 & 2 & 6,3 & \\
\hline \multicolumn{8}{|l|}{ Alimentação } \\
\hline Consideram saudável & 20 & 36,4 & 24 & 43,6 & 11 & 20,0 & \multirow{2}{*}{$0,042^{\dagger}$} \\
\hline Não consideram saudável & 25 & 62,5 & 10 & 25,0 & 5 & 12,5 & \\
\hline
\end{tabular}

Fonte: dados da pesquisa, 2016. *Pontos de corte para o Índice de Massa Corpórea = de 18,5-24,9 kg/m² (eutrofia), 25 a $29,9 \mathrm{~kg} / \mathrm{m}^{2}$ (sobrepeso) e $\geq 30 \mathrm{~kg} / \mathrm{m}^{2}$ (obesidade); 'Teste Qui-Quadrado de Pearson; 'Teste Exato de Fisher 
Tabela 4 - Associação dos domínios da Escala de Avaliação do Estresse nos Estudantes de Enfermagem com o Índice de Massa Corpórea. Porto Alegre, RS, Brasil, 2016. $n=95$ estudantes

\begin{tabular}{|c|c|c|c|c|c|c|c|}
\hline \multirow{3}{*}{ Domínios da Escala AEEE ${ }^{*}$} & \multicolumn{6}{|c|}{ Índice de Massa Corporal ${ }^{\dagger}$} & \multirow{3}{*}{$\mathbf{p}^{\ddagger}$} \\
\hline & \multicolumn{2}{|c|}{ Eutrofia } & \multicolumn{2}{|c|}{ Sobrepeso } & \multicolumn{2}{|c|}{ Obesidade } & \\
\hline & $\mathbf{n}$ & $\%$ & $\mathbf{n}$ & $\%$ & $\mathbf{n}$ & $\%$ & \\
\hline \multicolumn{8}{|l|}{ Realização de Atividades Práticas } \\
\hline Baixo nível de estresse & 14 & 45,2 & 11 & 35,5 & 6 & 19,4 & \multirow{4}{*}{0,680} \\
\hline Médio nível de estresse & 17 & 56,7 & 9 & 30,0 & 4 & 13,3 & \\
\hline Alto nível de estresse & 10 & 45,5 & 7 & 31,8 & 5 & 22,7 & \\
\hline Muito alto nível de estresse & 4 & 33,3 & 7 & 58,3 & 1 & 8,3 & \\
\hline \multicolumn{8}{|l|}{ Comunicação Profissional } \\
\hline Baixo nível de estresse & 22 & 53,7 & 10 & 24,4 & 9 & 22,0 & \multirow{4}{*}{0,341} \\
\hline Médio nível de estresse & 9 & 39,1 & 12 & 52,2 & 2 & 8,7 & \\
\hline Alto nível de estresse & 7 & 41,2 & 6 & 35,3 & 4 & 23,5 & \\
\hline Muito alto nível de estresse & 7 & 50,0 & 6 & 42,9 & 1 & 7,1 & \\
\hline \multicolumn{8}{|l|}{ Gerenciamento do Tempo } \\
\hline Baixo nível de estresse & 30 & 50,0 & 17 & 28,3 & 13 & 21,7 & \multirow{4}{*}{0,378} \\
\hline Médio nível de estresse & 5 & 45,5 & 6 & 54,5 & 0 & 0 & \\
\hline Alto nível de estresse & 7 & 38,9 & 9 & 50,0 & 2 & 11,1 & \\
\hline Muito alto nível de estresse & 3 & 50,0 & 2 & 33,3 & 1 & 16,7 & \\
\hline \multicolumn{8}{|l|}{ Ambiente } \\
\hline Baixo nível de estresse & 26 & 44,8 & 24 & 41,4 & 8 & 13,8 & \multirow{4}{*}{0,674} \\
\hline Médio nível de estresse & 8 & 47,1 & 6 & 35,3 & 3 & 17,6 & \\
\hline Alto nível de estresse & 5 & 62,5 & 1 & 12,5 & 2 & 25,0 & \\
\hline Muito alto nível de estresse & 6 & 50,0 & 3 & 25,0 & 3 & 25,0 & \\
\hline \multicolumn{8}{|l|}{ Formação Profissional } \\
\hline Baixo nível de estresse & 11 & 47,88 & 8 & 34,8 & 4 & 17,4 & \multirow{4}{*}{0,659} \\
\hline Médio nível de estresse & 6 & 66,7 & 2 & 22,2 & 1 & 11,1 & \\
\hline Alto nível de estresse & 13 & 46,4 & 8 & 28,6 & 7 & 25,0 & \\
\hline Muito alto nível de estresse & 15 & 42,9 & 16 & 45,7 & 4 & 11,4 & \\
\hline \multicolumn{8}{|l|}{ Atividade Teórica } \\
\hline Baixo nível de estresse & 22 & 52,4 & 14 & 33,3 & 6 & 14,3 & \multirow{4}{*}{0,465} \\
\hline Médio nível de estresse & 14 & 51,9 & 7 & 25,9 & 6 & 22,2 & \\
\hline Alto nível de estresse & 7 & 43,8 & 7 & 43,8 & 2 & 12,5 & \\
\hline Muito alto nível de estresse & 2 & 20,0 & 6 & 60,0 & 2 & 20,0 & \\
\hline
\end{tabular}

Fonte: dados da pesquisa, 2016. *AEEE = Avaliação de Estresse nos Estudantes de Enfermagem; ${ }^{\dagger}$ Pontos de corte para o Índice de Massa Corpórea = de $18,5-24,9 \mathrm{~kg} / \mathrm{m}^{2}$ (eutrofia), 25 a $29,9 \mathrm{~kg} / \mathrm{m}^{2}$ (sobrepeso) e $\geq 30 \mathrm{~kg} / \mathrm{m}^{2}$ (obesidade); ${ }^{\ddagger}$ Teste Exato de Fisher

\section{Discussão}

O perfil dos estudantes caracterizou-se por serem predominantemente mulheres, corroborando pesquisas brasileiras realizadas pelo Conselho Federal de Enfermagem que evidenciam composição majoritária de profissionais do sexo feminino $(87,2 \%)^{(16)}$. Também vão ao encontro de outros achados na literatura a faixa etária adulto jovem $(65,5 \%)$ e solteiros $(88,6 \%)^{(17)}$, além dos autorreferidos da cor branca $(78,9 \%)^{(18)}$.

Mais da metade dos estudantes desta pesquisa afirmam que realizam as principais refeições e não as trocam por lanches rápidos. Dentre as refeições que os estudantes mais realizam, destacam-se o almoço e o jantar. No entanto, menos da metade dos estudantes realizam o desjejum e a ceia. $\mathrm{O}$ almoço também foi a refeição realizada com mais frequência em pesquisa sobre hábitos de vida em estudantes de Enfermagem, em torno de quatro a sete vezes por semana $(73,4 \%)$ ${ }^{(19)}$. Este estudo também evidenciou que a frequência de lanches por semana variou de uma a três vezes $(45,8 \%)$ e quatro a sete vezes $(24,5 \%)$ pelos estudantes ${ }^{(19)}$.

O hábito de realizar outras atividades (assistir TV, usar o celular, por exemplo) enquanto fazem suas refeições parece recorrente na literatura. O uso do celular durante as refeições apareceu em 14\% dos estudantes, por necessidade de manterem-se mais tempo conectados $(39,5 \%)^{(20)}$. Dado preocupante, pois, 
ao fazê-lo, o estudante se priva de um momento muito importante que é o de saborear o alimento, de sentir a saciedade e não ficar aquém ou além das necessidades nutricionais.

Apesar de a classificação de estudantes com eutrofia possuir o maior percentual $(47,4 \%)$, a alteração de peso chama a atenção quando somados os percentuais do sobrepeso $(35,8 \%)$ e obesidade I e II $(16,8 \%)$, pois atingem $52,6 \%$ dos estudantes deste estudo. Esses achados superam as prevalências já identificadas, em que $26 \%$ dos estudantes de Enfermagem de uma universidade da Bahia estavam com sobrepeso e 4,5\%, com obesidade I e II ${ }^{(21)}$ e a prevalência combinada de sobrepeso/obesidade em $32,4 \%$ dos estudantes da saúde em duas universidades mexicanas ${ }^{(22)}$.

A 7 a Diretriz Brasileira de Hipertensão(12), destaca que o aumento de peso está diretamente relacionado ao aumento da PA. Neste estudo, quase $50 \%$ dos estudantes foram classificados com pré-hipertensão e $26,3 \%$ com hipertensão. As alterações de circunferência abdominal sinalizam para a deposição de gordura visceral, que é mais um fator importante a ser considerado na prevenção de agravos a saúde dos estudantes.

Passar por situações estressantes pode contribuir para alterações nos hábitos alimentares, dentre eles, o aumento da ingesta alimentar, o que predispõe para o sobrepeso e obesidade de forma significativa(22). Os estudantes deste estudo apresentaram alto (29,5\%) e muito alto nível de estresse $(36,8 \%)$ referente ao Domínio 5 da AEEE, que avalia questões da formação profissional. Outros estudos brasileiros também apresentaram maiores percentuais (em torno de $50 \%$ ) de estresse nesse domínio(23-24). Vários fatores podem estar relacionados, entre eles, o mercado de trabalho estar cada vez mais competitivo, exigindo dos profissionais da área da saúde mais especializações e conhecimentos teórico-práticos, além da experiência.

Ao contrário de outros estudos que evidenciam relação significativa entre estresse, sobrepeso e obesidade ${ }^{(5,22)}$, os achados deste estudo não evidenciaram diferenças estatísticas significativas entre a alteração ou não do peso dos estudantes, conforme a classificação do IMC, e os níveis de estresse avaliados pelos domínios da AEEE $(p>0,05)$. A relação significativa foi encontrada com algumas variáveis demográficas e de saúde.

Estudo que avaliou fatores de risco para doenças cardiovasculares em estudantes de Enfermagem associou IMC $\geq 25$ nas faixas etárias 21 a 30 anos de idade $^{(25)}$, diferente deste que não evidenciou diferença significativa entre os grupos avaliados $(p=0,06)$.

Ser do sexo masculino teve associação significativa ao IMC na faixa sobrepeso ( $p=0,03$ ) quando comparado às mulheres que foram classificadas na categoria com eutrofia. Em estudantes mexicanos, a prevalência de sobrepeso/obesidade também foi significativamente maior em homens $(38,5 \%)$ do que em mulheres $(30,5 \%)^{(22)}$. Dado contrário à realidade brasileira, pois segundo o site da ABESO, a prevalência maior de excesso de peso está no sexo feminino $(58,2 \% \times 55,6 \%)^{(26)}$, mas os percentuais são muito próximos. A variação de peso confirmou uma associação significativa esperada com o IMC ( $p<0,001)$, ou seja, o estudante com variação de peso $\leq 3,55 \mathrm{~kg}$ foi classificado com eutrofia $(71,1 \%)$ e aqueles com variação de peso $\geq 3,55 \mathrm{~kg}$ foram classificados com sobrepeso $(46,7 \%)$ e obesidade $(26,7 \%)$.

A circunferência abdominal também se associou ao IMC ( $p<0,001$ ), ou seja, maior percentual de estudantes com risco cardiovascular aumentado foi classificado em sobrepeso (69\%) e os estudantes com risco substancialmente aumentado apresentam classificação em obesidade (71,4\%). Sobre isso, não há consenso na literatura, pois estudo que comparou circunferência abdominal e IMC entre o primeiro e o último ano do curso de enfermagem não encontrou variação significativa desses indicadores; no entanto, $59,1 \%$ dos estudantes não tinham valores de circunferência abdominal adequados ${ }^{(21)}$, valor próximo ao deste estudo (52,6\%).

Esses achados são reforçados pelo fato de que os estudantes não consideram sua alimentação saudável e de não terem o hábito de praticar atividade física: muitos daqueles que não praticam atividade física foram classificados na categoria obesidade (27,9\%). Segundo a ABESO, o estilo de vida moderno favorece o ganho de peso. A necessidade de se realizar refeições em curto espaço de tempo, somado ao uso de celular durante as refeições, por exemplo, atrapalha os mecanismos de saciação. A interrupção da prática de esportes e a diminuição do gasto energético diário também são mecanismos influenciadores de ganho de peso, o que pode estar ligado à falta de tempo(2) e à dinâmica da vida acadêmica. Estudo que avalia a prática de atividade física entre estudantes de Enfermagem identificou $57,8 \%$ dos estudantes como sedentários ${ }^{(27)}$. A 7 a Diretriz de Hipertensão Brasileira relata que a inatividade física tem sido considerada um dos maiores problemas de saúde pública, por ser mais prevalente e ser a segunda causa de morte no mundo(12).

As manifestações físicas disso podem acontecer em cadeia, pois os estudantes classificados com hipertensão tiveram maior distribuição nas categorias sobrepeso $(54,2 \%)$ e obesidade (25\%). Dentre as recomendações da ABESO está que a redução de peso resulta na redução da pressão arterial, além da melhora de colesterol HDL (High Density Lipoprotein) e LDL (Low Density Lipoprotein), glicemia, entre outros. A mudança 
no comportamento é capaz de reduzir a pressão arterial e o risco de hipertensão em um ano(1).

Um estilo de vida saudável também auxilia na redução dos níveis de ansiedade em situações de estresse. Essas situações são prejudiciais e se mostraram significativas na relação entre sentir mais vontade de comer e sobrepeso $(39,7 \%)$, quando comparados aos classificados na categoria eutrofia que, mesmo em situação de estresse, sentem menos vontade de comer $(65,6 \% ; p=0,025)$. Esses resultados podem estar ligados à existência de uma associação entre sentir estresse e a sensação de melhora depois de desfrutar de alimentos ricos em calorias palatáveis, como açúcar e gordura. Essa postura pode se tornar hábito e, uma vez aprendida essa informação, as pessoas podem começar a usar o alimento para aliviar situações estressoras ${ }^{(1)}$. O consumo aumentado de alimentos em situações de ansiedade é recorrente na literatura(5,22).

Foram limitações do estudo: o delineamento transversal (viés da causalidade reversa); o peso corporal autorreferido no início da graduação (não havia dados coletados anteriormente sobre essa variável), e a diminuta existência de literaturas que associem fatores ligados ao estresse (Escala AEEE), sobrepeso e obesidade em estudantes de Enfermagem. Uma dificuldade quanto à Escala AEEE é a mesma não possuir um ponto de corte geral para classificar os estudantes em situação de estresse, pois a classificação ocorre por domínios. Outra dificuldade foi a de adesão à pesquisa por parte dos estudantes, fato atrelado a horários de aulas, falta de tempo, alguns relatos de constrangimento relacionado aos valores das medidas antropométricas e, principalmente, a verificação do peso. No entanto, foi atingido o número amostral mínimo para os achados serem representativos da população de estudantes.

\section{Conclusões}

$\mathrm{Na}$ população deste estudo, verificou-se que não há associação entre estresse, sobrepeso e obesidade. Mostram-se associadas de forma significativa ao sobrepeso e/ou obesidade ser estudante do sexo masculino, ter pressão arterial em níveis elevados, ter tido ganho de peso desde o início do curso, estar com a circunferência abdominal alterada, não realizar atividade física, comer mais em situações de estresse e ingerir alimentos não saudáveis.

Apesar das limitações e dificuldades apontadas na execução deste estudo, foi possível identificar alguns estressores para os estudantes de enfermagem, que podem subsidiar reflexões acerca de estratégias para a diminuição destes de forma a contribuir para a formação profissional do enfermeiro e a redução dos agravos relacionados ao sobrepeso e à obesidade. É importante que esse tema seja aprofundado e amplamente discutido em investigações futuras, para que o estudante atente para sua saúde e riscos que podem afetá-la. Como futuros enfermeiros, eles têm importante papel na promoção da saúde e prevenção de doenças, em especial as cardiovasculares e as psicossomáticas. Deste modo, o estudante, desde o início da graduação, também possui papel fundamental na promoção da sua saúde e na prevenção de agravos. Para isso, considerase importante que sejam desenvolvidas estratégias de promoção de saúde entre os estudantes da graduação, a fim de melhorar este panorama e minimizar os riscos futuros que podem ser causados pela obesidade.

\section{Referências}

1. Associação Brasileira para o Estudo da Obesidade e da Síndrome Metabólica. Diretrizes brasileiras de obesidade. [Internet]. 4ª ed. São Paulo: A Associação; 2016, 188p. [Acesso 2 jun, 2017]. Disponível em: http://www.abeso. org.br/uploads/downloads/92/57fccc403e5da.pdf

2. Ministério da Saúde (BR). Secretária de Atenção à saúde. Departamento de Atenção Básica. Cadernos de Atenção Básica - Obesidade. [Internet]. Brasília: Ministério da Saúde; 2006, 108p. [Acesso 2 jun, 2017]. Disponível em: http://189.28.128.100/dab/docs/ publicacoes/cadernos_ab/abcad12.pdf

3. Costa ALS, Polak C. Construction and validation of an instrument for the assessment of stress among nursing students (AEEE). Rev Esc Enferm USP. [Internet]. 2009 Dez [cited Jun 2, 2017];43(spe):1017-26. Available from: http://www.scielo.br/pdf/reeusp/v43nspe/a05v43ns.pdf

4. Chipas A, Cordrey D, Floyd D, Grubbs L, Miller S, Tyre B. Stress: perceptions, manifestations, and coping mechanisms of student registered nurse anesthetists. AANA J. [Internet]. 2012 Ago [cited Jun 2, 2017];80(4):49-55. Available from: https://www.aana. com/newsandjournal/Documents/stress-perc-manicop-mech-srnas-0812-pS49-S55.pdf

5. Borges KM, Figueiredo FWS, Souto RP. Night eating syndrome and emotional states in university students. J Hum Growth Dev. [Internet]. 2017 Dec [cited Jan 24, 2019]; 27(3):132-9. Available from: http://pepsic. bvsalud.org/pdf/rbcdh/v27n3/pt_11.pdf

6. Monteiro CFS, Freitas JFM, Ribeiro AAP. Stress in the academic daily: a nursing student view from the Federal University of Piauí - Brazil. Esc Anna Nery. [Internet]. 2007 Mar [cited Jun 2, 2017];11(1):66-72. Available from: http://www.scielo.br/pdf/ean/v11n1/v11n1a09.pdf 7. Rodrigues EOL, Marques DA, Lopes D Neto, Montesinos MJL, Oliveira ASA. Stressful situations and factors in students of nursing in clinical practice. 
Invest Educ Enferm. [Internet]. $2016 \mathrm{Apr}$ [cited Jun 2, 2017];34(1):211-20. Available from: http:// www.scielo.org.co/scielo.php?script=sci_arttext\&pid $=\mathrm{S} 0120-53072016000100023$

8. Vitela SC, Pacheco AE, Carlos ALS. Síndrome de burnout e estresse em graduandos de enfermagem. Rev Enferm Centro-Oeste Min. [Internet]. 2013 [Acesso 2 jun 2017];3(3):780-87. Disponível em: http://www. seer.ufsj.edu.br/index.php/recom/article/view/415

9. Silva VLS, Chiquito NC, Andrade RAPO, Brito MFP, Camelo SHH. Stress factors in the final year of undergraduate nursing: students' perceptions. Rev Enferm UERJ. [Internet]. 2011 Jan/Mar [cited Jun 2, 2017];19(1):121-6. Available from: http://www.facenf. uerj.br/v19n1/v19n1a20.pdf

10. Duarte FM, Almeida SDS, Martins KA. Away-fromhome food consumption of students from some courses in the health area of a private university. Mundo Saúde. [Internet]. 2013 Jul/Set [cited Jun 2, 2017];37(3):28898. Available from: http://bvsms.saude.gov.br/bvs/ artigos/mundo_saude/alimentacao_fora_domicilio_ universitarios_cursos.pdf

11. Ministério da Saúde (BR). Secretaria de Atenção à Saúde. Departamento de Atenção Básica. Orientações para avaliação de marcadores de consumo alimentar na atenção básica. [Internet]. Brasília: Ministério da Saúde; 2015, 33p. [Acesso 2 jun 2017]. Disponível em: http:// bvsms.saude.gov.br/bvs/publicacoes/marcadores_ consumo_alimentar_atencao_basica.pdf

12. Malachias MVB, Gomes MAN, Nobre F, Alessi A, Feitosa AD, Coelho EB. 7th Brazilian guideline of arterial hypertension: chapter 2 - Diagnosis and classification. Arq Bras Cardiol. [Internet]. 2016 Sep [cited Jun 2, 2017];107(supp3):7-13. Available from: http://www. scielo.br/scielo.php?script=sci_arttext\&pid=S0066782X2016004800007

13. World Health Organization. Physical status: the use and interpretation of anthropometry. [Internet] Geneva: World Health Organization; 1995. [cited Jun 2, 2017]. Available from: http://apps.who.int/iris/ bitstream/10665/37003/1/WHO_TRS_854.pdf

14. Associação Brasileira para o Estudo da Obesidade e da Síndrome Metabólica. Diretrizes brasileiras de obesidade 2009/2010. [Internet]. 3a ed. Itapevi: AC Farmacêutica; 2009. 85p. [Acesso 2 jun 2017]. Disponível em: http://www.abeso.org.br/pdf/diretrizes_ brasileiras_obesidade_2009_2010_1.pdf

15. International Diabetes Federation (BE). The IDF consensus worldwide definition of the metabolic syndrome. [Internet]. Brussels: International Diabetes Federation; 2006. 24p. [cited Jun 2, 2017]. Available from: https://www.idf.org/e-library/consensus-statements/ 60-idfconsensus-worldwide-definitionof-the-metabolicsyndrome

16. Conselho Federal de Enfermagem, Comissão de Business Intelligence. Produto 2: Análise de dados dos profissionais de enfermagem existentes nos Conselhos Regionais. [Internet]. Brasília: Departamento de Tecnologia da Informação; 2011. 71p. [Acesso 2 jun 2017]. Disponível em: http://www.cofen.gov.br/wpcontent/uploads/2012/03/pesquisaprofissionais.pdf

17. Kawakame PM, Miyadahira AM. Quality of life of undergraduate students in nursing. Rev Esc Enferm USP. [Internet]. 2005 Jun [cited Jun 2, 2017]:39(2)164-

72. Available from: http://www.scielo.br/pdf/reeusp/ v39n2/06.pdf

18. Machado $\mathrm{MH}$, Aguiar W Filho, Lacerda WF, Oliveira $\mathrm{E}$, Lemos $\mathrm{W}$, Wermelinger $\mathrm{M}$, et al. Características gerais da enfermagem: perfil sócio demográfico. Enferm Foco. [Internet]. 2015 [Acesso 2 jun 2017];6(1/4):11-7. Disponível em: http://revista.cofen.gov.br/index.php/ enfermagem/article/download/686/296

19. Alves EF. Estilo de vida de estudantes de graduação em enfermagem de uma instituição do Sul do Brasil. Rev CPAQV. [Internet]. 2011 [Acesso 2 jun 2017]:3(1)1-13. Disponível em: http://www.cpaqv.org/revista/CPAQV/ ojs-2.3.7/index.php?journal=CPAQV\&page $=$ article\&op $=$ view\&path $\% 5 B \% 5 D=31$

20. Silva FC, Vizzotto MM. Profile of the college student user technologies. Psicol inF. [Internet]. 2013 [cited Jun 2, 2017]:17(17)39-55. Available from: https://www. metodista.br/revistas/revistas-ims/index.php/PINFOR/ article/viewFile/4549/3748

21. Pires CGS, Mussi FC. Excess weight in nursing junior and senior undergraduate students. Esc Anna Nery Rev Enferm. [Internet]. 2016 Oct [cited Jun 2, 2017]:20(4):e20160098. Available from: http://www.scielo.br/scielo.php?pid=S1414$81452016000400215 \&$ script $=$ sci_arttext\&tlng=en

22. Blandón DAS, León TC, Durango MPP, TejadaTayabas LM, Lucio AGP. Ansiedad, depresión y actividad física asociados a sobrepeso/obesidad en estudiantes de dos universidades mexicanas. Hacia Promoc Salud. [Internet]. 2016 Jul/Dez [Acceso 24 jan 2019]: 21(2):99-113. Disponible en: http://www.scielo.org.co/ pdf/hpsal/v21n2/v21n2a08.pdf

23. Costa MA, Oliveira JL, Souza VS, Inoue KC, Reis GA, Matsuda LM. The association between stress and sociodemographic variable on nursing student of an institution in Parana. Rev Enferm UFJF. [Internet]. 2016 Jun [cited Jun 2, 2017]:2(1):9-19. Available from: https://enfermagem.ufjf.emnuvens.com.br/ enfermagem/article/view/67/36

24. Souza FA, Paiano M. Challenges and difficulties faced by nurses at the beginning of their career. REME 
Rev Min Enferm. [Internet]. 2011 Jun [cited Jun 2, 2017];15(2):267-73. Available from: http://www.reme. org.br/content/imagebank/pdf/v15n2a16.pdf

25. 25. Alves A, Marques IR. Associated risk factors for coronary heart disease among nursing students. Rev Bras Enferm. [Internet]. 2009 Dec [cited Jun 2, 2017];62(6):883-8. Available from: http://www.scielo. br/pdf/reben/v62n6/a13v62n6.pdf

26. Associação Brasileira para o Estudo da Obesidade e da Síndrome Metabólica. [Internet] Itapevi: AC Farmacêutica [Acesso 2 jun 2017]. Quase 60\% dos brasileiros estão acima do peso, revela IBGE. Disponível em: http://www. abeso.org.br/noticia/quase-60-dos-brasileiros-estaoacima-do-peso-revela-pesquisa-do-ibge 27. Pires CG, Mussi FC, Cerqueira BB, Pitanga FJ, Silva DO. Physical activity practice among undergraduate students in nursing. Acta Paul Enferm. [Internet]. 2013 [cited Jun 2, 2017]:26(5):436-43. Available from: http://www.scielo.br/pdf/ape/v26n5/a06v26n5.pdf 